\title{
Physical and Dosimetric Aspects of the Iridium-Knife
}

\author{
Yiannis Roussakis $^{\star t}$ and Georgios Anagnostopoulos ${ }^{\dagger}$ \\ Department of Medical Physics, German Oncology Center, Limassol, Cyprus
}

The three-dimensional iridium-192 ( ${ }^{192}$ Ir) high-dose-rate (HDR) brachytherapy manifests itself as a high-precision, hypofractionated, dose-escalating, minimally invasive method in the armamentarium of contemporary radiation oncology clinical applications. In this study, the physical aspects of the ${ }^{192}$ Ir radionuclide are presented. Its dosimetric application in HDR brachytherapy for different anatomical sites (prostate, gynecological malignancies, liver, and intrathoracic tumors) as well as the corresponding dosimetric comparison with the stereotactic body radiation therapy (SBRT) techniques based on a representative selection of dosimetric publications is reviewed and illustrated.

\section{OPEN ACCESS}

Edited by:

Valdir Carlos Colussi, University Hospitals Cleveland Medical

Center, United States

Reviewed by: Wei Zhao,

Beihang University, China Andrei Fodor, IRCCS San Raffaele Scientific Institute, Italy

*Correspondence:

Yiannis Roussakis yiannis.roussakis@goc.com.cy

${ }^{\dagger}$ These authors have contributed equally to this work

Specialty section: This article was submitted to Radiation Oncology, a section of the journal Frontiers in Oncology

Received: 21 June 2021 Accepted: 19 October 2021 Published: 09 November 2021

Citation:

Roussakis $Y$ and Anagnostopoulos $G$ (2021) Physical and Dosimetric

Aspects of the Iridium-Knife.

Front. Oncol. 11:728452. doi: 10.3389/fonc.2021.728452
Keywords: iridium knife, brachytherapy, dosimetry, high-dose-rate (HDR) brachytherapy, stereotactic ablative radiotherapy (SABR), stereotactic body radiation therapy (SBRT), stereotactic radiosurgery (SRS), iridium-192

\section{INTRODUCTION}

Iridium-192 ( ${ }^{192}$ Ir) has been extensively used for high-dose-rate (HDR) brachytherapy (BT) for more than four decades in the remote afterloading BT systems. Its physical properties are presented in Table 1 (1-5). Its medium energy range of $\mathrm{X}$ - and gamma-rays (mean energy: $370 \mathrm{keV}$ ) in its energy spectrum and its beneficial reference air kerma rate (RAKR) of $0.1091 \mu \mathrm{Gym}^{2} \mathrm{~h}^{-1} \mathrm{MBq}^{-1}$ in comparison with other HDR source radionuclides such as ${ }^{60} \mathrm{Co}$ (energy: 1.173 and $1.332 \mathrm{MeV}$, RAKR: $0.308 \mu \mathrm{Gym}^{2} \mathrm{~h}^{-1} \mathrm{MBq}^{-1}$ ) have always required definitively less shielding than any external beam radiation modality. The tenth value layer (TVL) for ${ }^{192} \mathrm{Ir}$ is 16 and $152 \mathrm{~mm}$ for lead and concrete, respectively, while for ${ }^{60} \mathrm{Co}$, the TVL is 41 and $218 \mathrm{~mm}$ for lead and concrete, respectively (4). This enables the construction of ${ }^{192}$ Ir HDR BT treatment rooms in a more cost-effective way and storage of the source inside the remote afterloader an easier task. The half-life of ${ }^{192} \mathrm{Ir}$ is 73.81 days, enabling a relatively economic application of this radionuclide in remote afterloading HDR systems for radiation therapy, by implementing four source exchanges per year, in order to maintain the HDR of greater or equal to $12 \mathrm{~Gy} / \mathrm{h}$. Its main advantage is especially the high specific activity $\left(340 \mathrm{GBqmg}^{-1}\right)$, which makes feasible the manufacturing and the distribution of high-activity ${ }^{192}$ Ir miniaturized size sources (370-480 GBq). The typical length of an encapsulated ${ }^{192}$ Ir HDR BT source is in the order of 5-11 mm, and the typical outer diameter lies in the order of $0.8-1 \mathrm{~mm}$. For distances from the source center, $r$, greater than the double of the ${ }^{192}$ Ir active core length ( $\mathrm{r} \geq 2 \mathrm{~L} \approx 8 \mathrm{~mm}$ ), the source can be considered a point source in its dosimetry $(2,3)$, and the dose rate distribution in the periphery of the source-hence the dose rate in the planning target volume (PTV) and in the surrounding healthy tissue-is determined by the inverse square law $\left(\mathrm{r}^{-2}\right)$. The immediate contact of the implanted catheter/applicator inside the PTV, where the miniaturized ${ }^{192} \mathrm{Ir}$ source is remotely driven, along with the predominance of the inverse square law of a single source in its dwell position, facilitates the characteristic steep dose fall-off, which in comparison with a single megavoltage external X-ray or even a proton beam (with the characteristic Bragg peak) is unbeatable.

The characteristic dosimetric property of the ${ }^{192}$ Ir BT source with its sharp dose gradient justifies reasonably the name "Iridium-Knife" in correspondence to the high-precision, steep-dose gradient 
TABLE 1 | Physical characteristics of ${ }^{192}$ Ir radionuclide and ${ }^{192}$ Ir sources used in brachytherapy (1-4).

\begin{tabular}{|c|c|}
\hline \multicolumn{2}{|l|}{${ }^{192} \mathrm{Ir}$} \\
\hline Half-life (days) & 73.81 \\
\hline Type of disintegration & $\begin{array}{c}\beta^{-} \text {(95.1\%), Electron Capture } \\
(4.9 \%)\end{array}$ \\
\hline Maximum x-ray energy [keV] & 78.6 \\
\hline Gamma energy range $[\mathrm{keV}]$ & $110.4-1,378.2$ \\
\hline Mean gamma ray energy $[\mathrm{keV}]$ & 370 \\
\hline $\begin{array}{l}\text { Air kerma rate constant, } \Gamma_{\delta}\left[\mu \mathrm{Gy} \mathrm{m} \mathrm{m}^{2} \mathrm{~h}^{-1}\right. \\
\left.\mathrm{MBq}^{-1}\right]\end{array}$ & 0.1091 \\
\hline Specific activity $\left[\mathrm{GBq} \mathrm{mg}^{-1}\right]$ & 341.0 \\
\hline Range of active ${ }^{192}$ Ir source core length [mm] & $3.5-10.0$ \\
\hline $\begin{array}{l}\text { Range of active }{ }^{192} \text { Ir source core diameter } \\
{[\mathrm{mm}]}\end{array}$ & $0.34-0.70$ \\
\hline Tenth value layer (TVL) in lead [mm] & 16 \\
\hline Tenth value layer (TVL) in concrete [mm] & 152 \\
\hline
\end{tabular}

d, days; keV, kilo electron-volt; $\mu$ Gy, micro-Gray; MBq, mega-Becquerel; GBq, gigaBecquerel; $m m$, millimeter; $m g$, milligram.

Linear Accelerator (LINAC)-based multi-leaf collimator and the robotic radiosurgery device-based external-beam radiotherapy, which are also known as X-knife (6) and Cyberknife (7), respectively.

The purpose of this mini-review is to illustrate the capability of the three-dimensional (3D) HDR BT with ${ }^{192}$ Ir for the delivery of conformal dose coverage of the PTV by concomitant dose escalation inside them and the steep dose fall-off in their periphery, acting thus as another precision dose knife, due to its physical properties. Furthermore, by reviewing representative published data for four different anatomical sites, a dosimetric comparison with the stereotactic body radiation therapy (SBRT) method is presented and the advantages as well as disadvantages of the ${ }^{192}$ Ir HDR BT are highlighted.

\section{MATERIALS AND METHODS}

In this article, we review dosimetric studies for prostate, liver, endometrium, and intrathoracic malignancies either clinically performed with HDR BT and afterward virtually planned with SBRT or vice versa (8-15). The dosimetric comparison for PTV coverage, organs at risk (OARs) dose sparing, plan conformity, dose heterogeneity, dose fall-off sharpness, and low dose spill are presented with the aid of dose-volume and physical quantities presented in Table 2. The clinical evaluation and the medical follow-up of the patients subjected to these radiotherapy modalities are beyond the scope of this article.

\section{REVIEW OF REPRESENTATIVE DOSIMETRIC STUDIES}

\section{Prostate}

For high- and intermediate-risk prostate cancer patients, a small number of planning studies have been carried out for the comparison of the established prostate HDR BT with the SBRT modality (8-10). It should be mentioned that the SBRT in the prostate was initially termed "virtual HDR" because of the similar characteristics to HDR BT, namely, hypofractionation and high dose per fraction administration.

In the first study (8), the patients were treated with ${ }^{192}$ Ir HDR BT with transrectal ultrasound (TRUS)-guided plastic needle insertion with a dose of $4 \times 9.5$ Gy as monotherapy. Following the recommendations of the American Brachytherapy Society, the dose parameters applied in the optimization phase were for the urethra $\mathrm{D}_{\max }$ less than $120 \%$ of the prescribed dose (PD), for rectum $\mathrm{D}_{\max }$ less than $100 \%$ of the $\mathrm{PD}$ and $\mathrm{D}_{2 \mathrm{ccm}}$ less than $70 \%$ of the PD, for the bladder $\mathrm{D}_{2 \mathrm{ccm}}$ less than $75 \%$ of the $\mathrm{PD}$, and for the PTV coverage V100\% greater than $90 \%$. Spratt et al. used two approaches to dosimetrically compare the HDR dosimetry with the virtual SBRT plans. The first was the "normal tissueprioritized" plan, where the primary goal was to maintain the OAR constraints by simultaneously trying to maximize target coverage, and the second approach was the "PTV-prioritized" plan, where the coverage of the PTV was the primary goal by allowing the constraints for the OARS to be violated in an as minimal as possible extent.

The dosimetric comparison results of the "normal tissueprioritized" plans are presented in Table 3 [Table 1 (8)] and reveal that all dose metrics for the rectum and the bladder were lower for the HDR BT, but only the rectal $D_{\max }$ reached statistical significance, representative of the sharper dose falloff outside of the PTV for the Iridium-Knife. As expected, dose metrics for the urethra were always higher for the HDR BT in comparison with SBRT due to the predominant inhomogeneity of the dose distributions around the source dwell positions inside the PTV and in the proximity of the urethra, a characteristic that is evident with the dose escalation feature and the resulting value of V200\% for the HDR BT inside the PTV, a value that cannot be reached by SBRT.

For the case of "PTV-prioritized" plans, where an attempt was made for SBRT to match the PTV V200\% of the HDR BT by relaxing the OAR constraints, the PTV V150\% was significantly higher $(67 \%)$ for SBRT than for the HDR BT plan (40\%) $(p=$ 0.045). Furthermore, rectum $D_{\max }$ and $D_{\text {mean }}$ for the SBRT were $111 \%$ and $33 \%$, respectively, while in HDR BT, they were significantly lower $94 \%(p=0.045)$ and $27 \%(p=0.028)$, respectively, thus violating the dosimetric constraints for rectum. Additionally, the bladder and urethral doses were higher for SBRT in this approach compared to HDR BT, without showing statistical significance. Another important finding of this study is that, for this plan approach, the surrounding healthy tissue total body dose was significantly greater for SBRT than for HDR BT $(\mathrm{V} 10 \%=$ $2,206 \mathrm{~cm}^{3}$ for SBRT vs. V10\% $=1,250 \mathrm{~cm}^{3}$ for HDR BT, $p=0.01$ ), implying that the dose escalation with SBRT inside the prostate PTV to approach HDR BT comes with a cost of increased dose spillage in the surrounding healthy tissue.

In the second study (9), six consecutive prostate patients have been treated HDR BT with a dose prescription of $45.5 \mathrm{~Gy}$ in seven fractions. Metallic needles were placed with real-time TRUS guidance, and the plan was created on a CT image acquired after needle insertion. The clinical target volume (CTV) comprised 
TABLE 2 | Dose-volume and physical quantities used for the dosimetric assessment of the radiation therapy modalities.

Dose-volume parameters

Definition or equation describing the quantity

Commonly used dose volume quantities and other plan quality factors

\begin{tabular}{|c|c|c|}
\hline \multicolumn{3}{|c|}{ PTV } \\
\hline VX\% & The volume of the PTV receiving X\% of the prescribed dose (PD) & $\begin{array}{l}\text { V100\%, V95\%, V90\%, V150\%, V200\%, } \\
\text { V105\% }\end{array}$ \\
\hline VXGy & The volume of the PTV receiving X Gy of dose & \\
\hline $\mathrm{DA} \%$ & The dose received by the A\% of the PTV & D95\%, D99\%, D100\% \\
\hline$D_{\min }$ & The minimum dose within the PTV as percentage of the PD & \\
\hline $\mathrm{D}_{\max }$ & $\begin{array}{l}\text { The maximum dose within the PTV as percentage of the PD (the same applies for the } \\
\text { volume of the OARs) }\end{array}$ & \\
\hline Conformity Index (Cl) & $V_{\text {PTV PD }} V_{\text {PTV }}\left(V_{\text {PTV PD }}\right.$ : volume of the PTV covered by the PD and $V_{\text {PTV: volume of PTV) }}$ & $\begin{array}{l}\mathrm{Cl} \text { values close to } 1.0 \text { indicate better } \\
\text { conformity of the PD to the PTV }\end{array}$ \\
\hline Conformation number (CN) & $\begin{array}{l}\left(V_{P T V} P D\right)^{2} N_{P T V} \times V_{P D}\left(V_{P T V} P D: \text { volume of the PTV covered by the PD and } V_{P T V}:\right. \\
\text { volume of PTV, } V_{P D} \text { : volume of the prescribed dose) }\end{array}$ & \\
\hline \multicolumn{3}{|c|}{ OARs } \\
\hline WY\% & The volume of the OAR receiving Y\% of the prescribed dose (PD) & V20\%, V40\%, V60\%, V80\%, V100\% \\
\hline VYGy & The volume of the OAR receiving $Y$ Gy of dose & \\
\hline $\mathrm{DB} \%$ & The dose received by the $\mathrm{B} \%$ of the OAR volume & D10\%, D20\% \\
\hline $\mathrm{D}_{\mathrm{yccm}}$ & The dose received by y cubic centimeters of the OARs & $\begin{array}{l}D_{0.1 \mathrm{ccm}}, D_{0.5 \mathrm{ccm}}, D_{1 \mathrm{ccm}}, D_{2 \mathrm{ccm}}, D_{5 \mathrm{ccm}} \\
D_{10 \mathrm{ccm}}, D_{1,000 \mathrm{ccm}}\end{array}$ \\
\hline $\begin{array}{l}\text { Dmean } \\
\text { Healthy Tissue Conformity Index } \\
\text { (HTCl) }\end{array}$ & $\begin{array}{l}\text { Mean dose received by the OAR } \\
\left(V_{\text {PTV PD }}\right)^{2} N_{\text {PTV }} \times V_{P D} \text { (it takes into account the irradiation of healthy tissue beyond the } \\
\text { PTV) }\end{array}$ & \\
\hline \multicolumn{3}{|c|}{ Conformality and dose fall-off gradient indices } \\
\hline Conformal Index (COIN) & $\begin{array}{l}\mathrm{CN} \times \Pi_{i}^{N}\left(1-\frac{V_{i, P D}}{V_{i}}\right) \\
\left(\mathrm{CN} \text { : conformation number, } \mathrm{N} \text { is the number of OARs under consideration, } V_{i, P D} \text { is the }\right. \\
\text { volume of the i-th OAR covered by the PD and } V_{i} \text { is the volume of the } \mathrm{i} \text {-th } \mathrm{OAR} \text { ) }\end{array}$ & $\begin{array}{l}\text { Each of the COIN component would be } \\
\text { ideally equal to } 1.0\end{array}$ \\
\hline High dose spillage (HDS) & $\begin{array}{l}\frac{V_{105 \% \text { for (body-PTV) }}}{V_{P T V}} \text { (it is the ratio of the volume outside of the PTV receiving } 105 \% \text { of } \\
\text { the PD to the volume of the PTV }\end{array}$ & $\begin{array}{l}\text { Any higher dose than } 105 \% \text { of the PD } \\
\text { should not be found outside the delineated } \\
\text { PTV }\end{array}$ \\
\hline $\mathrm{R}_{50 \%}$ & The ratio of the volume receiving $50 \%$ of the PD to the volume of the PTV & \\
\hline $\mathrm{D}_{2 \mathrm{~cm}}$ & $\begin{array}{l}\text { Maximum relative dose } 2.0 \mathrm{~cm} \text { beyond the PTV in any direction as percentage of the } \\
\text { PD }\end{array}$ & \\
\hline V20\%-V80\% & $\begin{array}{l}\text { Healthy tissue volume outside the PTV receiving between } 80 \% \text { and } 20 \% \text { of the PD } \\
\text { expressed in } \mathrm{cm}^{3}\end{array}$ & \\
\hline
\end{tabular}

PTV, planning target volume; $P D$, prescribed dose; OAR, organ at risk; ccm, cubic centimeter.

the prostate gland plus $5 \mathrm{~mm}$ in all directions to cover possible extracapsular extensions, except for the posterior rectal margin, which varied from 2 to $5 \mathrm{~mm}$ depending on the distance from the rectal wall. The planning goal in that study was to achieve a V $100 \%$ of partially lower than $100 \%$ (because V100\% $=100 \%$ is impossible due to the interference of the metallic needle positions with the urethra shape in the anterior ventral part of the prostate that cannot be intersected, thus leaving a cold spot in that area), with urethra $\mathrm{D}_{\max }$ lower than $150 \%$ of the $\mathrm{PD}$ and rectum $\mathrm{D}_{\max }$ lower than $100 \%$ of the PD.

The virtual SBRT (CyberKnife) planning was performed with the CT images and structures from the HDR BT plan, except for the

TABLE 3 | The results of the dosimetric comparison of the normal tissue-prioritized plans from the study of Spratt et al. (8) [courtesy of Spratt et al. (8), reproduced with permission].

\begin{tabular}{|c|c|c|c|c|c|}
\hline Dosimetric parameter & SBRT plans & \pm Standard Deviation & HDR plans & \pm Standard Deviation & $p$-value \\
\hline PTV V100\% & $93.08 \%$ & 3.20 & $93.78 \%$ & 1.78 & Non-significant \\
\hline PTV V150\% & $42.86 \%$ & 7.70 & $40.32 \%$ & 6.47 & Non-significant \\
\hline PTV V200\% & $0.00 \%$ & 0.00 & $15.18 \%$ & 3.05 & 0.00 \\
\hline Rectum $D_{\max }$ & $99.42 \%$ & 2.79 & $94.24 \%$ & 5.24 & 0.05 \\
\hline Rectum $D_{2 \mathrm{ccm}}$ & $71.14 \%$ & 4.78 & $60.84 \%$ & 5.90 & 0.07 \\
\hline Rectum $D_{\text {mean }}$ & $28.43 \%$ & 4.00 & $27.12 \%$ & 4.03 & Non-significant \\
\hline Bladder $D_{\max }$ & $110.06 \%$ & 9.92 & $104.17 \%$ & 30.05 & Non-significant \\
\hline Bladder $D_{2 \mathrm{ccm}}$ & $78.78 \%$ & 6.41 & $58.30 \%$ & 9.58 & 0.08 \\
\hline Urethra $D_{\max }$ & $115.80 \%$ & 5.40 & $119.28 \%$ & 3.98 & Non-significant \\
\hline Urethra $D_{1 \mathrm{ccm}}$ & $75.17 \%$ & 29.72 & $87.72 \%$ & 12.87 & Non-significant \\
\hline Urethra $D_{\text {mean }}$ & $84.83 \%$ & 13.11 & $95.04 \%$ & 9.96 & 0.08 \\
\hline
\end{tabular}

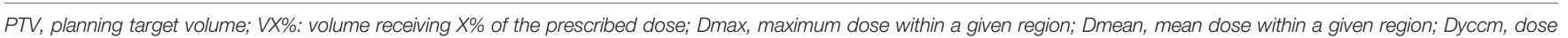
received by y cubic centimeters of a given region. 
PTV that was adjusted with an additional margin of $2 \mathrm{~mm}$. The SBRT dosimetric constraints were for the PTV: dose within $100 \%$ and $150 \%$ of the $\mathrm{PD}, \mathrm{D}_{\max }$ for rectum, urethra, and bladder to be less or equal to $100 \%$ of the PD. For each SBRT plan, the most common prescription method of D95\% was used as the prescription dose to perform the dosimetric comparison of SBRT with HDR BT.

The results for dose volume parameters of the PTV [Table 1 (9)] revealed that, in terms of dose escalation inside the PTV, the HDR BT is significantly better than the SBRT modality. However, the $\mathrm{D} 99 \%$, the $\mathrm{D} 100 \%$, and the $\mathrm{V} 100 \%$ SBRT values are higher than the corresponding ones of HDR BT due to the inherent limitation of the HDR BT to dosimetrically fully cover with the PD the anterior part of the PTV, where the urethra is located, because the urethra should not be penetrated with needles. Considering the dose received by lower volume fractions of the PTV, such as the D90\%, HDR BT performed significantly better than SBRT. Moreover, the V125\%, the V150\%, and the V200\% values of HDR BT are significantly higher than the ones of SBRT, and this dose escalation could lead to a better outcome (16).

The dosimetric comparison for the rectum revealed that HDR BT had a higher $\mathrm{D}_{\max }$ and a steeper dose fall-off inside the rectum than SBRT in the intermediate to high dose range. The average $D_{1 \mathrm{ccm}}, D_{2 \mathrm{ccm}}, D_{5 \mathrm{ccm}}, D_{10 \mathrm{ccm}}$, and $\mathrm{D}_{20 \mathrm{ccm}}$ were significantly lower for HDR BT by 5.1, 7.1, 7.6, 6.1, and 2.4 Gy, respectively. Moreover, the average V40\%-100\% values were also significantly lower in HDR BT, even though they were relatively small $\left(1.6-4.5 \mathrm{~cm}^{3}\right)$. For the urethra, the HDR BT was inferior to SBRT with statistical significance. The average $\mathrm{D}_{0.1 \mathrm{ccm}}$, $\mathrm{D}_{0.2 \mathrm{ccm}}, \mathrm{D}_{0.5 \mathrm{ccm}}, \mathrm{D} 10 \%$, and $\mathrm{D} 20 \%$ were considerably higher than for SBRT by $12.6,8.9,4.5,10.2$, and 7.0 Gy, respectively. For the bladder, HDR BT resulted in higher doses than the SBRT modality, with no statistical significance however, except for the $\mathrm{D}_{0.5 \mathrm{ccm}}$, which was equal to $96.2 \mathrm{~Gy}$ for HDR BT and $51.1 \mathrm{~Gy}$ for SBRT ( $p=0.02$ ). This might be attributed to the fact that, for the HDR BT, source dwell positions of catheters inside the bladder pouch were activated in order to be able to cover the anterior cranial part of the prostate PTV with the $100 \%$ of the PD, resulting in very high doses in the bladder.

In the third study (10), 15 patients were treated with SBRT (CyberKnife) with a dose prescription of $35 \mathrm{~Gy}$ in five fractions. TRUS-based fiducial marker implantation was performed at least 5 days before treatment planning CT acquisition, and a TRUS dataset was acquired immediately before implantation. For the SBRT plans, GTV was defined as the prostate on MRI in low risk and prostate with base of seminal vesicles $(1 \mathrm{~cm}$ proximal) for all other patients. CTV was generated by uniform expansion of the CTV by $2 \mathrm{~mm}$, while PTV was generated by anisotropic expansion of the CTV ( $1 \mathrm{~mm}$ posterior and $3 \mathrm{~mm}$ in all other directions). The planning goal was to achieve V100\% of more than 95\% for the PTV and V37.5Gy of more than 95\% for the GTV. The dosimetric constraints for rectum $D_{\max }$ were lower than 38 Gy, and V36Gy, V29Gy, and V18Gy encompassing less than 1,15 , and $25 \mathrm{~cm}^{3}$, respectively. For bladder, the $D_{\max }$ was lower than $38 \mathrm{~Gy}$, with V36Gy less than $10 \mathrm{~cm}^{3}$ and V18Gy covering less than $40 \%$ of its volume. For urethra, Dmax was lower than $44 \mathrm{~Gy}$ and V44Gy less than $20 \%$ of its volume.
The virtual HDR BT plans were created on the aforementioned TRUS images with a dose prescription of $35 \mathrm{~Gy}$ in a single fraction in order to allow direct dosimetric comparison to the clinical SBRT plan. The PTV encompassed the entire prostate gland without margins. The planning goal was to achieve D90\% of more than $100 \%$ and D150\% less than 35\% for the PTV. The dosimetric constraints for rectum $\mathrm{D}_{0.1 \mathrm{ccm}}$ were less than $80 \%$ of $\mathrm{PD}$ for rectum and bladder and less than $120 \%$ of the PD for urethra.

The dosimetric comparison for the prostate (i.e., SBRT GTV or HDR BT PTV) showed that the dose delivered to $98 \%$ of the volume, the V35Gy and V37.5Gy were significantly higher for SBRT compared to HDR BT plans, while V42Gy and V52.5Gy were significantly higher for HDR BT compared to SBRT plans. The maximum dose to the rectum and bladder was significantly lower, while the maximum dose to the urethra was significantly higher in HDR BT plans compared to SBRT plans.

\section{Liver}

A small number of studies thus far attempted to dosimetrically compare brachytherapy plans against SBRT plans in the treatment of primary or secondary malignancies of the liver $(11,12)$.

Pennington et al. (11) investigated the differences of brachytherapy and SBRT plans in a retrospective study of 10 patients with liver metastasis, originally treated with SBRT, and for which virtual ${ }^{192}$ Ir HDR BT plans were created. Both plans were designed to deliver five fractions of 12 Gy to the same PTV, while no precise information is given for the generation of this volume. The stated HDR BT planning goal was to match the PTV receiving $100 \%$ of the PD to the SBRT plan. In terms of target coverage, they found that the mean PTV V100\% was comparable between HDR BT and SBRT $(94.1 \%$ vs. $93.9 \%, p=$ 0.8 ), while mean PTV V150\% was significantly higher in HDR BT plan (63.6\% vs. $0 \%)$, revealing significant dose escalation. On the other hand, significantly lower minimum dose as a percentage of the prescription dose within the PTV was exposed for HDR BT compared to SBRT plans (66\% vs. $88 \%$, $p=0.0002)$. They found no statistically significant differences ( $p=0.109)$ in dose fall-off as estimated by R50\%. The authors concluded that HDR BT can achieve higher target dose, similar dose to OARs but potentially lower target coverage in comparison with SBRT (11). A limitation to this study is the retrospective virtual HDR BT planning of the data, coupled with the fact that the same PTV was irradiated as for the SBRT plans. This is typically not the case in clinical practice where the CTV is considered to be the PTV in HDR BT treatments, since due to the fact that needles are fairly stable within the tumor, the uncertainties addressed by the PTV expansion are deemed negligible. Therefore, even though it is a fact that in HDR BT clinical practice, it is often difficult for the prescription dose to cover the entirety of the PTV, a fairer comparison of the two modalities would compare the coverage of the prescription dose for the respective PTV employed for each modality.

In a recent study, Hass et al. (12) performed a similar comparison of HDR BT vs. SBRT plans for liver lesions while addressing the aforementioned limitations of previous studies. 
In their article, 85 patients previously treated for liver malignancies with HDR BT were used, and for each, a retrospective virtual SBRT plan was generated. The GTV was generated based on contrast-enhanced breath-hold CT scanning with a 3-5-mm margin for the generation of the CTV, depending on the visualization quality of the GTV. No additional margin was added for HDR BT planning, and therefore, CTV = PTV for this case. In contrast, a margin of $5 \mathrm{~mm}$ in lateral direction and $10 \mathrm{~mm}$ along the craniocaudal axis was added to the GTV for the generation of the SBRT-specific PTVs. The prescription dose for both treatments was the same for both plans (15 or 20 Gy in one fraction, depending on type of tumor) and was prescribed for both treatment modalities to be $99.9 \%$ of the PTV. The same OAR constraints were employed. The dosimetric results of this investigation are summarized in Table 4. They revealed significantly better coverage PTV D99.9\% by the prescribed HDR BT plans compared to SBRT plans in both the $15 \mathrm{~Gy}$ $(p<0.001)$ and $20 \mathrm{~Gy}(p=0.003)$ groups. Similarly, mean PTV D90\% was significantly higher in the HDR BT plans in both the $15-\mathrm{Gy}(p<0.001)$ and $20 \mathrm{~Gy}(p<0.001)$ groups. Regarding the exposure of the remaining liver volume, the study found no statistically significant differences in V5Gy in the 15 Gy group ( $p=0.095$ ), but statistical significance was found in the $20 \mathrm{~Gy}$ group ( $p=0.001$ ) in favor of the HDR BT plans. The authors concluded that HDR BT revealed superior outcomes both in terms of target coverage (PTV D99.9\% and D90\%) and exposure to the remaining healthy liver. They also discussed further advantages to each of the examined modalities. Namely, HDR BT allows for irradiation of larger liver tumors (SBRT is typically limited to sizes with diameter less than $4-6 \mathrm{~cm}$ ) and more centrally located tumors (for which SBRT is typically avoided) while also being less affected by uncertainties related to respiratory breathing motion. On the other hand, HDR BT is a minimally invasive procedure while SBRT is non-invasive. The authors point out some limitations in their study. The main one is the fact that their planning procedure for SBRT was aiming for a relatively homogeneous dose distribution inside the target, avoiding central dose escalation. This would typically lead to a shallower dose gradient than would otherwise be achieved if the authors allowed the SBRT treatment planning optimizer the flexibility to have a higher dose in the central region of the PTV. Therefore, steeper dose fall-off would be expected with current SBRT planning strategies that allow up to $125 \%-140 \%$ of the prescription dose within the target.

\section{Gynecological Malignancies}

A number of investigators performed retrospective studies to dosimetrically compare HDR BT and SBRT sequential boost in the treatment of gynecologic malignancies, and two representative investigations are discussed herein $(13,14)$.

Georg et al. (13) attempted a comparison of HDR BT against SBRT in cervical cancer using high-tech techniques for both methods. Nine patients with locally advanced cervical cancer that were previously treated with HDR BT boost under MRI guidance were employed. Additional SBRT plans were created with stepand-shoot intensity-modulated radiotherapy (IMRT) and intensity-modulated proton therapy (IMPT) for each patient. Both SBRT treatment plans were created under the assumptions that (a) MRI-guided beam delivery was available, (b) dedicated applicator for cervix immobilization would be used, (c) online adaptive planning would be employed, and (d) sufficient patient immobilization would exist. For HDR BT plans, the PTV was taken to be the same as the CTV, while for the SBRT plans, two PTV scenarios were created, with 5- and 3-mm expansion of the GTV for the generation of the SBRT-specific PTV. For all cases, the plans created to deliver four fractions of 7 Gy. HDR BT plans were prescribed such that the prescription dose would fully cover the PTV, while SBRT plans aimed to deliver the highest possible dose to the GTV and PTVs [intermediate-risk PTV (IR-PTV) and high-risk PTV (HR-PTV)] while maintaining the same OAR DVH parameters previously achieved by the HDR BT plans. Since the SBRT planning constraints adopted the HDR BTachieved OAR doses, these were very similar. The authors revealed that for IMRT plans limited to the HDR BT-achieved OAR constraints, GTV or PTV D90\% was in general lower than the respective HDR BT plans. For IMPT plans, D90\% was mostly similar or lower to that of HDR BT plans. When ratios of volumes receiving 3 and 3.5 Gy were compared, IMRT plans revealed volumes twice as large, and volumes receiving 5 Gy were 1.5 times as large as HDR BT plans, regardless of the PTV margin size. Volumes receiving 7 Gy were on average smaller in IMRT than HDR BT plans. For IMPT, volumes receiving 3, 3.5, and 5 Gy were approximately 1.5 times larger compared to HDR BT plans. They concluded that for image-guided cervical cancer sequential boost treatments, both IMRT and IMPT seem to be inferior to HDR BT (13).

Yildirim et al. (14) recently performed a similar retrospective dosimetric study comparing HDR BT plans against SBRT plans designed with VMAT technique on a conventional linear

TABLE 4 | The dosimetric results (mean \pm standard deviation) of the different brachytherapy (BT) and stereotactic body radiotherapy (SBRT) from the study of Hass et al. (12) [courtesy of Hass et al. (12), reproduced with permission].

\begin{tabular}{|c|c|c|c|c|c|c|c|c|c|}
\hline & \multicolumn{3}{|c|}{ Overall } & \multicolumn{3}{|c|}{ 15-Gy prescription dose } & \multicolumn{3}{|c|}{ 20-Gy prescription dose } \\
\hline & BT & SBRT & $p$ & BT & SBRT & $p$ & BT & SBRT & $p$ \\
\hline $\mathrm{D}_{90 \%}[\mathrm{~Gy}]$ & $27.9 \pm 0.4$ & $19.5 \pm 0.3$ & $<0.001$ & $24.3 \pm 0.8$ & $16.5 \pm 0.3$ & $<0.001$ & $29.2 \pm 0.4$ & $20.6 \pm 0.3$ & $<0.001$ \\
\hline$D_{99.9 \%}[\mathrm{~Gy}]$ & $18.8 \pm 0.4$ & $16.8 \pm 0.4$ & $<0.001$ & $16.0 \pm 0.4$ & $14.7 \pm 0.4$ & 0.003 & $19.9 \pm 0.4$ & $17.5 \pm 0.5$ & $<0.001$ \\
\hline Liver $V_{5 G y}\left[\mathrm{~cm}^{3}\right]$ & $593 \pm 36$ & $671 \pm 33$ & $<0.001$ & $544 \pm 65$ & $607 \pm 71$ & 0.098 & $611 \pm 43$ & $694 \pm 43$ & 0.674 \\
\hline Liver $V_{5 G y}[\%]$ & $39.5 \pm 2.0$ & $43.6 \pm 1.7$ & 0.001 & $33.3 \pm 2.7$ & $37.3 \pm 3.0$ & 0.095 & $41.8 \pm 2.5$ & $45.9 \pm 2.0$ & 0.977 \\
\hline
\end{tabular}

$B T$, brachytherapy; SBRT, stereotactic body radiotherapy; DA\%, dose received by A\% of a given region; VXGy, the volume of a given region receiving X Gy of dose. 
accelerator (Axesse, Elekta AB, Stockholm, Sweden) and on a HiArt Tomotherapy system (TomoTherapy Inc., Madison, WI). Twelve patients with early-stage endometrial cancer previously treated postoperatively with HDR BT were included and for which SBRT plans were retrospectively created with the two abovementioned technologies. The PTV was defined as a uniform 3D 5-mm expansion of the cylinder volume at the upper $3-5 \mathrm{~mm}$ of the vagina and was the same for all planning cases. The PD was $25 \mathrm{~Gy}$ in five fractions. The goal of the plan in both HDR BT and SBRT cases was that $95 \%$ of the PTV to receive at least $95 \%$ of the $\mathrm{PD}$ and $100 \%$ of the target volume to receive at least $90 \%$ of the $\mathrm{PD}$. The authors reported total coverage of target volumes with $150 \%-250 \%$ of the PD for the HDR BT plans. The mean PTV D98\% was $24.71 \pm 0.36$ Gy for the TomoTherapy plans and $24.42 \pm 0.38$ Gy for the VMAT plans, i.e., slightly lower than the prescription dose and were deemed adequate. Regarding the OAR doses, bladder $\mathrm{D}_{2 \mathrm{cc}}$ was found significantly lower in HDR BT plans than in VMAT and TomoTherapy plans, with no significant differences observed in the rectum $\mathrm{D}_{2 \mathrm{cc}}$ between the three plans. The authors concluded that their investigation demonstrated comparable PTV coverage between SBRT and HDR BT plans with lower inhomogeneity in the SBRT compared to the HDR BT plans and claimed that this study showed the feasibility of using SBRT as an alternative to HDR BT in endometrial cancer patients treated postoperatively (14). A limitation to this study was the use of the same PTV in both SBRT and HDR BT plans, created with the expansion of the $\mathrm{CTV}$, even though it is unlikely to be performed clinically due to the inherent positioning uncertainties associated with external beam radiotherapy treatments.

As a result of a growing recent utilization of SBRT treatments instead of the established HDR BT option in clinical settings, Gill et al. (17) utilized the United States National Cancer Database for the evaluation of the potential impact of SBRT usage in cervical cancer treatments. A total of 7,654 patients with stage IIB-IVA cervical cancer for which boost modality information was available were used. Of these patients, 90.3\% received HDR BT and the rest received IMRT or SBRT. It was observed that from 2004 to 2011, the use of brachytherapy decreased from $96.7 \%$ to $86.1 \%$, while use of IMRT and SBRT increased from 3.3\% to $13.9 \%(p<0.01)$. The comparative survival analysis between the two modalities revealed that IMRT or SBRT boost resulted in inferior overall survival (hazard ratio, 1.86; 95\% confidence interval, 1.35-2.55; $p=0.01$ ) when compared to HDR BT boost. The authors concluded that the increased use of IMRT and SBRT techniques for delivering the boost dose in cervical cancer patients with the concurrent apparent increase in mortality risk should raise concerns when deciding on the use of these modalities over HDR BT outside of clinical trials.

Addressing the concern raised by the analysis by Gill et al. (17), the Society of Gynecologic Oncology and the American Brachytherapy Society recently published a review article (18) that includes a discussion on the comparison of brachytherapy with IMRT or SBRT. They conclude with the statement "(...) conformal external beam therapies such as IMRT or SBRT should not be used as alternatives to brachytherapy in patients undergoing primary curative-intent radiation therapy for cervical cancer".

\section{Intrathoracic Malignancies}

In the study of Milickovic et al. (15), five patients with intrathoracic malignancies (IMs) of different sizes, that received X knife SBRT, were selected for ${ }^{192}$ Ir HDR BT comparative plan analysis. These patients were planned with the treatment planning system (TPS) Oncentra MasterPlan v4.5 (Elekta, Veenendaal, Netherlands) with 9-10 non-coplanar 6 MV X-ray beams delivering doses from 5 Gy up to 20 Gy per fraction. In order to ensure healthy tissue sparing, the planned dose encompassing the PTV was set equal to $80 \%$ of the isocenter dose. By using the same structures (PTV and OARs), they generated HDR BT virtual plans with the Oncentra Brachytherapy v4.5 TPS, with the planning goal to achieve the same PTV with the PD of the SBRT and the aid of the hybrid inverse optimization algorithm installed in the TPS (19). The number of virtual catheters for the BRT plans ranged from 6 to 10 , and the number of activated dwell positions pro $\mathrm{cm}^{3}$ was 3 . For the comparative dosimetric analysis, DVH was calculated and the paired Student's $t$-test with a $p$-value of 0.05 as significance threshold was applied.

The results of this study show a significantly better PTV coverage $(p=0.030)$ with the HDR BT for the V100\% $(93.04 \%$ of the PD) vs. the SBRT ( $88.94 \%$ of the PTV), while the other VX\% and DX\% parameters revealed no significant benefit of the HDR BT over SBRT, although the values are always greater for the HDR BT in comparison to the corresponding SBRT ones, except for the $\mathrm{D}_{\text {min }}$. The value of V150\% for HDR BT equals to 24.67, while for SBRT is 0.0 , as expected, indicating the ability of HDR BT for significant dose escalation inside the PTV $\left(p=4.84 \times 10^{-4}\right)$.

The CI, HTCI, CN, and COIN parameters did not show significant differences. For the high-dose spillage, there was no significant difference shown for both treatment methods because the amount of healthy tissue receiving doses more than $105 \%$ of the PD is low. For the intermediate-dose spillage, the $\mathrm{R}_{50 \%}$ of the HDR BT treatment plans was significantly ( $p=$ $0.002)$ better (2.47) than the corresponding one of the SBRT (21.14), $\mathrm{D}_{2 \mathrm{~cm}}$ was significantly higher for the SBRT treatment plans than for the HDR BT $(p<0.001)$, and V20\%-V80\% was significantly in favor of HDR BT $(p=0.003)$. In the same study, it has been illustrated that this volume difference favors the usage of HDR BT with increasing PTV. The comparison of the dose indices of the OARs exhibited no statistically significant difference of the two methods, except for the $D_{\max }$ in the spinal cord, where for HDR BT, the average value equals to $12.85 \mathrm{~Gy} \pm$ $7.42 \%$ and for the SBRT equals to $21.14 \mathrm{~Gy} \pm 5.72 \%$ of the PD $(p=0.022)$.

The study of Milickovic et al. (15) showed that SBRT is not dosimetrically superior to HDR BT and furthermore that HDR BT can deliver higher dose escalation inside the PTV in intrathoracic cases. Regarding larger lesions, where SBRT might not be able to deliver a sufficient dose due to normal tissue dose constraints, HDR BT is shown to manifest itself as a therapeutic option, considering the dosimetric aspect, although 
the results of the study might differ, if real implants are considered, because catheter placement alters the PTV anatomy.

\section{Cerebral Malignancies}

Milickovic et al. (15) also selected five patients with cerebral malignancies (recurrent glioblastoma multiform) who received X knife stereotactic radiosurgery (SRS) for retrospective dosimetric comparison against ${ }^{192}$ Ir HDR BT. SRS and HDR BT plans were generated with the same considerations described above for the intrathoracic malignancies in the same publication.

The results show that similar coverage was achieved by the two modalities (HDR BT: 96.09\%, SRS: 94.73\%) with no statistically significant differences $(p=0.227)$. As mentioned above, VX\% and DX\% parameters revealed no statistically significant differences besides V150\%, which was significantly higher for HDR BT compared to SRS plans.

The HTCI, CN, and COIN parameters did not show significant differences besides CI, which was significantly better $(p=0.026)$ for HDR BT than for SBRT. No significant differences were observed for $R_{50 \%}$, while $D_{2 \mathrm{~cm}}$ and $V 20 \%-V 80 \%$ revealed significant differences in favor of $\operatorname{HDR} \mathrm{BT}(p=0.032$ and $p=0.035$, respectively). Dose incidence for OARs did not reveal any significant differences either between the two treatment modalities.
Milickovic et al. (15) conclude that HDR BT is at least as good as SRS in cerebral malignancies-when the anatomical position of the target allows needle placement-the steep dose fall-off was shown to be sharper in HDR BT plans, while intermediate- and low-dose spillage was as good or significantly lower in HDR BT plans.

\section{DISCUSSION AND CONCLUSIONS}

This article deals with the illustration of the basic ${ }^{192}$ Ir physical properties, which justify its steep dose fall-off in its application for the interventional radiation oncology. Additionally, it reviews representative dosimetric comparison studies of the SBRT vs. the HDR BT modality (summary in Table 5). The clinical comparison and analysis review are beyond the scope of this article, as it is also for the assessment of the impact of the uncertainties each of the two modalities is linked to.

For prostate, two groups $(8,9)$ performed dosimetric comparison of real HDR BT plans with virtual SBRT plans, while the third group (10) compared real SBRT plans against virtual HDR BT plans. In general, SBRT revealed significantly better D100\% and D99\% than HDR BT due to the inherent limitation of HDR BT to fully cover areas inside the PTV that are anteriorly intersecting with

TABLE 5 | Summary of study year, original clinical plan, quantitative treatment plan analysis metrics employed, and number of patient cases utilized for the studies mentioned in current review.

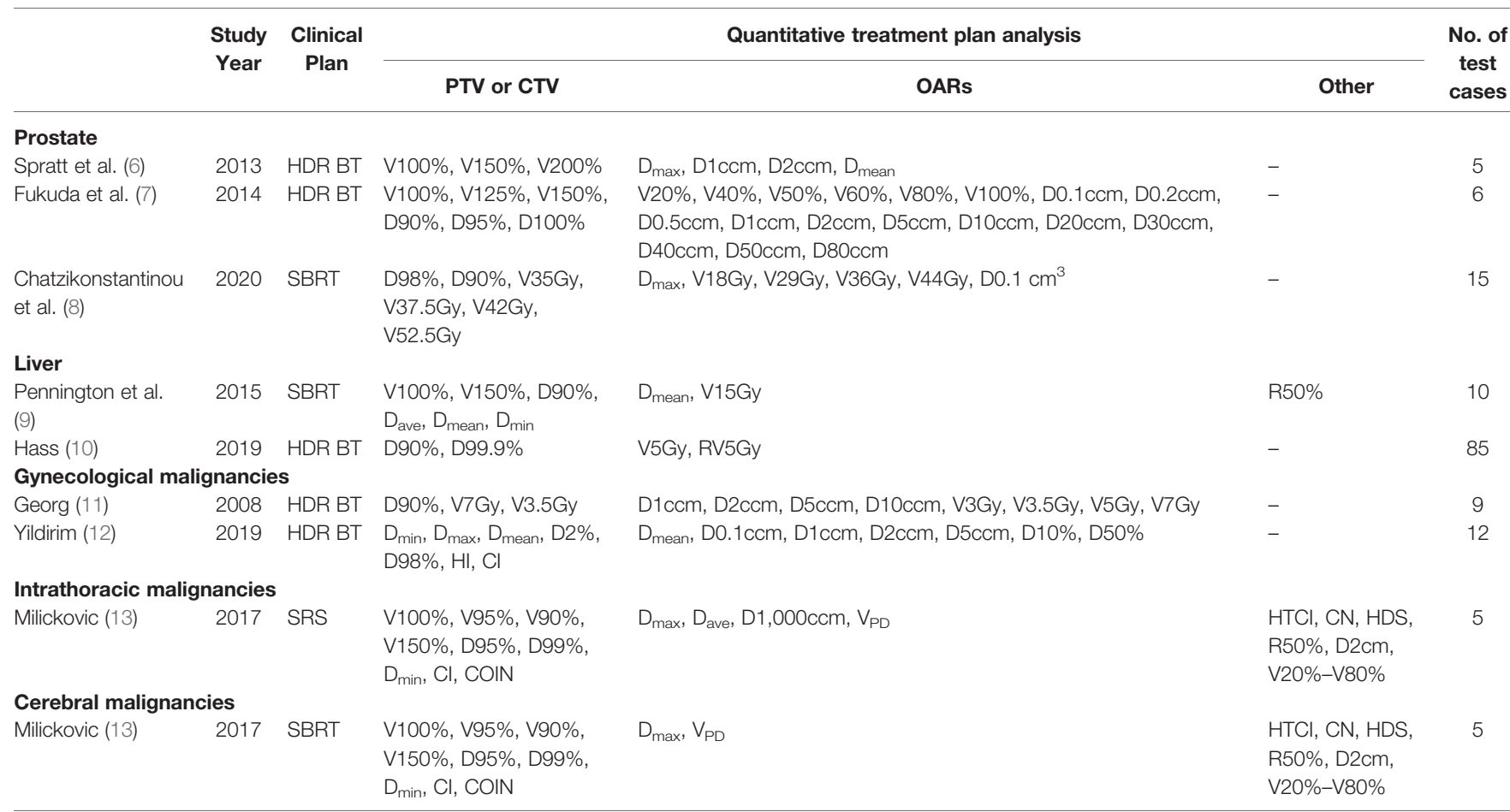

PTV, planning target volume; CTV, clinical target volume; OARs, organs at risk; HDR BT, high-dose-rate brachytherapy; SBRT, stereotactic body radiotherapy; SRS, stereotactic radiosurgery; $V x \%$, volume of a given region receiving $x \%$ of the prescribed dose; $V_{P D}$, volume of a given region receiving the prescribed dose; $D x c c m$, minimal radiation dose for the most irradiated volume of $x \mathrm{~cm}^{3}$ of a given region; $D x \%$, minimal radiation dose for the most irradiated volume of $x \%$ of a given region; $D_{\text {min, }}$, minimum dose in a given region; $D_{\text {max }}$ maximum dose in a given region; $D_{a v e}$, average dose in a given region; $R V x G y$, relative volume of organ receiving $x$ Gy (in \%); $H I$, homogeneity index; Cl, conformity index; $H T C l$, healthy tissue conformity index; CN, conformation number; COIN, conformal index; HDS, high-dose spillage; R50\%, the ratio of the volume receiving $50 \%$ of the prescribed dose to PTV; Dxcm, maximum relative dose $x \mathrm{~cm}$ beyond the PTV in any direction; V20\%-V80\%, healthy tissue volume (tissue outside the PTV) receiving between $80 \%$ and $20 \%$ of the prescribed dose. 


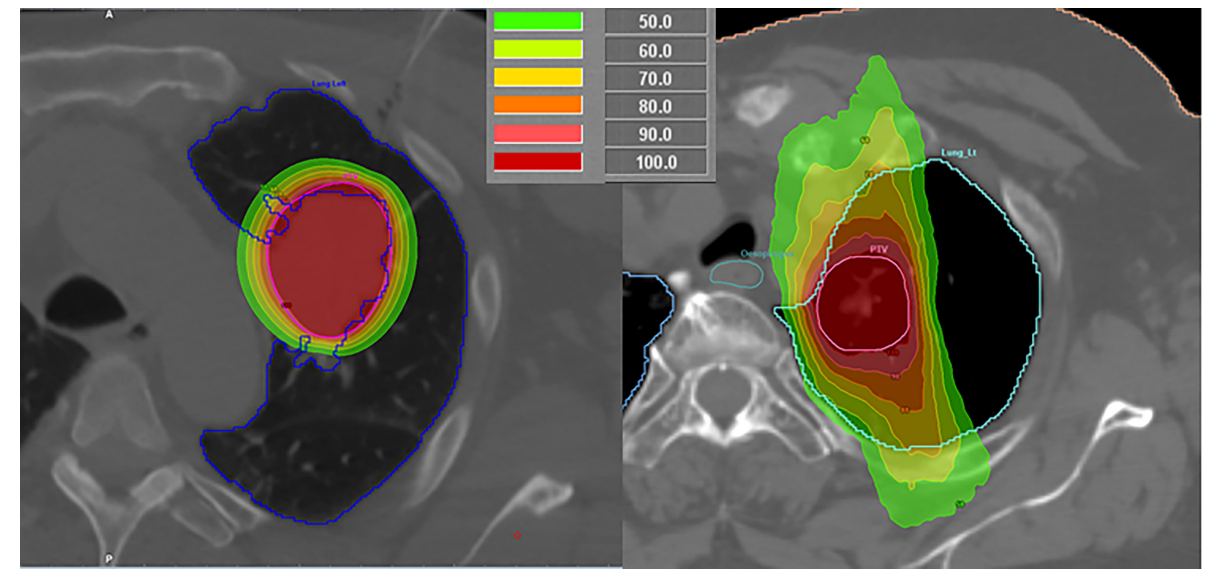

FIGURE 1 | Comparison of a high-dose-rate brachytherapy (left) and stereotactic body radiotherapy (right) plans for lung malignancies in different patients.

the urethra. In terms of dose escalation, HDR BT showed significantly higher average V150\% and V200\% values compared to SBRT. HDR BT performed significantly better for the dose to the rectum and the bladder but not for the dose inside the urethra in the first (8) and third (10) studies. In the second study (7), HDR BT performed significantly better for rectum but worse for bladder and urethra than SBRT. Both modalities have their advantages and disadvantages regarding the PTV coverage and the sparing of the OARs, with only one dosimetric feature that might be clinically decisive in future clinical trials, namely, the dose escalation inside the prostate PTV, which HDR BT can provide.

In liver malignancies, Pennington et al. (11) dosimetrically compared SBRT and virtual HDR BT, treating the same PTV with both techniques, and concluded that HDR BT could achieve higher target dose, similar dose to OARs, but potentially lower minimum dose to the target. Hass et al. (12) concluded that HDR BT revealed superior outcomes in terms of both target coverage and exposure to the remaining healthy liver.

In gynecologic malignancies, Yildirim et al. (14) used the same PTV to generate plans for both modalities and demonstrated slightly lower but comparable PTV coverage for SBRT plans compared to HDR BT plans and similar doses to the OARs, except the bladder $D_{2 c c}$, which was found significantly lower in HDR BT plans. Georg et al. (13) used different PTVs for each modality and attempted to use the high-tech techniques each modality has to offer and revealed inferior performance for SBRT in terms of both target coverage and steepness of dose falloff. A retrospective survival analysis based on the United States National Cancer Database (17), triggered by an apparent growing clinical implementation of SBRT replacing HDR BT, revealed an apparent increase in mortality risk associated with an increased use of IMRT and SBRT techniques, instead of HDR $\mathrm{BT}$, for delivering the boost dose in cervical cancer patients.

For intrathoracic malignancies, SBRT has been shown by Milickovic et al. (15) to be non-superior to HDR BT in its dosimetric aspect. Especially with regard to larger PTVs, HDR BT holds value in covering locations, where SBRT is unable to deliver sufficient dose because of normal tissue dose constraints, because the low-dose regions around the PTV increase much faster for SBRT than in the case of the HDR BT (example in Figure 1). The dose fall-off sharpness of the " ${ }^{~} 192$ Ir-knife" is moreover capable of boosting dose inside the PTV with its inherent inhomogeneous dose distribution, leading to a dose escalation that cannot be reached by SBRT for intrathoracic PTVs.

In cerebral malignancies, Milickovic et al. (15) demonstrated that HDR BT can be at least as good as SRS when the anatomical location of the target allows for needle placement. Significant dose escalation with significantly lower intermediate- and low-dose spillage was also demonstrated to be achievable with HDR BT plans.

While the ${ }^{192}$ Ir BT (Iridium-Knife) with its invasive nature requires additional expertise, experience, and logistics in comparison with external beam radiotherapy ablative options (SBRT, SRS), it manifests itself as an extremely "sharp knife" in terms of delivering higher dose gradients and simultaneously reducing the delivered dose to adjacent OARs. Furthermore, the integral dose delivered to the patient can be significantly reduced in comparison to SBRT and SRS treatment options, according to the studies presented herein. Its ability to significantly escalate dose inside the target region may have a key role in the era of personalized treatments, aiding the investigations toward optimal combinations of ablative radiation doses combined with immunotherapy (20) or present a practical solution for tumors with radioresistant hypoxic regions (21). HDR BT and external beam ablative options should be considered equivalent and complementary radiotherapy techniques and chosen based on their merits for each anatomical region and individual patient and tumor characteristics.

\section{AUTHOR CONTRIBUTIONS}

YR and GA conceived and designed the review, collected and analyzed the representative studies from the literature, and wrote and approved the submitted version. 


\section{REFERENCES}

1. Baltas D, Sakelliou L, Zamboglou N. The Physics of Modern Brachytherapy for Oncology. Boca Raton: CRC Press, Taylor \& Francis Group (2007), ISBN: .

2. Rivard MJ, Coursey BM, DeWerd LA, Hanson WF, Huq MS, Ibbott GS, et al. Update of AAPM Task Group No. 43 Report: A Revised AAPM Protocol for Brachytherapy Dose Calculations. Med Phys (2004) 31:633-74. doi: 10.1118/ 1.1646040

3. Perez-Calatayud J, Ballester F, Das RK, Dewerd LA, Ibbott GS, Meigooni AS, et al. Dose Calculation for Photon-Emitting Brachytherapy Sources With Average Energy Higher Than 50kev: Report of the AAPM and ESTRO. Med Phys (2012) 39:2904-29. doi: 10.1118/1.3703892

4. International atomic energy agency. Radiation Protection in Design of Radiotherapy Facilities, Safety Reports Series No. 47. Vienna: IAEA (2006).

5. Strohmaier S, Zwierzchowski G. Comparison of 60Co and 192Ir Sources in HDR Brachytherapy. J Contemp Brachyther (2011) 3(4):199-208. doi: $10.5114 /$ jcb.2011.26471

6. Wang L, Li J, Paskalev K, Hoban P, Luo W, Chen L, et al. Commissioning and Quality Assurance of a Commercial Stereotactic Treatment-Planning System for Extracranial IMRT. J Appl Clin Med Phys (2006) 7:21-34. doi: 10.1120/ jacmp.v7i1.2125

7. Bibault JE, Prevost B, Dansin E, Mirabel X, Lacornerie T, Lartigau E. ImageGuided Robotic Stereotactic Radiation Therapy With Fiducial-Free Tumor Tracking for Lung Cancer. Radiat Oncol (2012) 7:102. doi: 10.1186/1748717X-7-102

8. Spratt DE, Scala LM, Folkert M, Voros L, Cohen GN, Happersett L, et al. A Comparative Dosimetric Analysis of Virtual Stereotactic Body Radiotherapy to High-Dose-Rate Monotherapy for Intermediate-Risk Prostate Cancer. Brachytherapy (2013) 12(5):428-33. doi: 10.1016/j.brachy.2013.03.003

9. Fukuda S, Seo Y, Shiomi H, Yamada Y, Ogata T, Morimoto M, et al. Dosimetry Analyses Comparing High-Dose-Rate Brachytherapy, Administered as Monotherapy for Localized Prostate Cancer, With Stereotactic Body Radiation Therapy Simulated Using CyberKnife. J Radiat Res (2014) 55(6):1114-21. doi: 10.1093/jrr/rru048

10. Chatzikonstantinou G, Keller C, Scherf C, Bathen B, Kohn J, Tselis N. RealWord Dosimetric Comparison Between CyberKnife SBRT and HDR Brachytherapy for the Treatment of Prostate Cancer. Brachytherapy (2020) 20(1):44-9. doi: 10.1016/j.brachy.2020.07.011

11. Pennington JD, Park SJ, Abgaryan N, Banerjee R, Lee PP, Loh C, et al. Dosimetric Comparison of Brachyablation and Stereotactic Ablative Body Radiotherapy in the Treatment of Liver Metastasis. Brachytherapy (2015) 14:537-42. doi: 10.1016/j.brachy.2015.04.002

12. Hass P, Mohnike K, Kropf S, Brunner TB, Walke M, Albers D, et al. Comparative Analysis Between Interstitial Brachytherapy and Stereotactic Body Irradiation for Local Ablation in Liver Malignancies. Brachytherapy (2019) 18(6):823-8. doi: 10.1016/j.brachy.2019.08.003

13. Georg D, Kirisits C, Hillbrand M, Dimopoulos J, Potter R. Image-Guided Radiotherapy for Cervix Cancer: High-Tech External Beam Therapy Versus
High-Tech Brachytherapy. Int J Radiat Oncol Biol Phys (2008) 71(4):1272-8. doi: 10.1016/j.jijrobp.2008.03.032

14. Yildirim BA, Dolek Y, Guler OC, Arslan G, Onal C. Dosimetric Comparison of Vaginal Vault Brachytherapy vs Applicator-Guided Stereotactic Body Radiotherapy With Volumetric Modulated Arc Therapy and Helical Tomotherapy for Endometrium Cancer Patients. Med Dosim (2019) 44 (4):332-8. doi: 10.1016/j.meddos.2018.11.005

15. Milickovic N, Tselis N, Karagiannis E, Ferentinos K, Zamboglou N. Iridium Knife: Another Knife in Radiation Oncology. Brachytherapy (2017) 16 (4):884-92. doi: 10.1016/j.brachy.2017.03.005

16. Kuban DA, Tucker SL, Dong L, Starkschall G, Huang EH, Cheung MR, et al. Long-Term Results of the M.D. Anderson Randomized Dose-Escalation Trial for Prostate Cancer. Int J Radiat Oncol Biol Phys (2008) 70:1003-7. doi: 10.1016/j.ijrobp.2007.06.054

17. Gill BS, Lin JF, Krivak TC, Sukumvanich P, Laskey RA, Ross MS, et al. National Cancer Data Base Analysis of Radiation Therapy Consolidation Modality for Cervical Cancer: The Impact of New Technological Advancements. Int J Radiat Oncol Biol Phys (2014) 90(5):1083-90. doi: 10.1016/j.ijrobp.2014.07.017

18. Holschneider CH, Petereit DG, Chu C, Hsu IC, Ioffe YJ, Klopp AH, et al. Brachytherapy: A Critical Component of Primary Radiation Therapy for Cervical Cancer. Brachytherapy (2019) 152(3):540-7. doi: 10.1016/j.brachy.2018.11.009

19. Karabis A, Giannouli S, Baltas D. HIPO: A Hybrid Inverse Treatment Planning Optimisation Algorithm in HDR Brachytherapy. Radiother Oncol (2005) 76:S29. doi: 10.1016/S0167-8140(05)81018-7

20. Filatenkov A, Baker J, Mueller AMS, Kenkel J, Ahn GO, Dutt S, et al. Ablative Tumor Radiation can Change the Tumor Immune Cell Microenvironment to Induce Durable Complete Remissions. Clin Cancer Res (2015) 21(16):372739. doi: 10.1158/1078-0432.CCR-14-2824

21. Horsman M, Overaard J. The Impact of Hypoxia and its Modification of the Outcome of Radiotherapy. J Radiat Res (2016) 57(Suppl 1):i90-8. doi: $10.1093 /$ jrr/rrw007

Conflict of Interest: The authors declare that the research was conducted in the absence of any commercial or financial relationships that could be construed as a potential conflict of interest.

Publisher's Note: All claims expressed in this article are solely those of the authors and do not necessarily represent those of their affiliated organizations, or those of the publisher, the editors and the reviewers. Any product that may be evaluated in this article, or claim that may be made by its manufacturer, is not guaranteed or endorsed by the publisher.

Copyright (๑) 2021 Roussakis and Anagnostopoulos. This is an open-access article distributed under the terms of the Creative Commons Attribution License (CC BY). The use, distribution or reproduction in other forums is permitted, provided the original author(s) and the copyright owner(s) are credited and that the original publication in this journal is cited, in accordance with accepted academic practice. No use, distribution or reproduction is permitted which does not comply with these terms. 